\title{
Predictive value of microparticle-associated tissue factor activity for permeability glycoprotein-mediated multidrug resistance in cancer
}

\author{
ANTONIO ANGELINI ${ }^{1,2}$, SEBASTIANO MISCIA ${ }^{1,2}$, MARIA ANTONIETTA CENTURIONE ${ }^{3}$, \\ ROBERTA DI PIETRO $^{1 *}$ and LUCIA CENTURIONE ${ }^{1^{*}}$ \\ ${ }^{1}$ Department of Medicine and Aging Science, School of Medicine and Health Science; ${ }^{2}$ Research Center on Aging \\ and Translational Medicine (Ce.S.I Met), 'G. d'Annunzio' University of Chieti-Pescara; ${ }^{3}$ Institute of \\ Molecular Genetics, National Research Council - Pavia, Chieti Unit, I-66013 Chieti, Italy
}

Received March 18, 2016; Accepted July 7, 2016

DOI: $10.3892 / \mathrm{ol} .2016 .5105$

\begin{abstract}
Multidrug resistance (MDR) protein 1, which is also known as permeability glycoprotein (Pgp), and tissue factor (TF) are recurrently overexpressed on the surface of cancer cells, likely in response to stimuli such as chemotherapy. Microparticles (MPs) released from cancer cells into the bloodstream express tumour markers on their surface that may be useful as predictive biomarkers for evaluating disease progression. The present study measured the level of TF/factor VII (FVII)-dependent coagulation of MPs isolated from the plasma of cancer patients with various tumours, who were undergoing chemotherapy. Furthermore, Pgp expression on the surface of MPs was evaluated by immunohistochemistry. A total of 50 cancer patients, as well as 10 healthy volunteers, were enrolled in the present study. MP-associated TF/FVII-dependent coagulation pathways were evaluated as the effect of an anti-FVII antibody on the time to thrombin generation, as compared with controls treated with saline. The significantly lengthened times of coagulation [obtained in 20/50 samples $(36.5 \pm 16 \%)$ after treatment with anti-FVIIa when compared with controls] suggest the presence of TF activity is associated with circulating MPs. Furthermore, the $20 \mathrm{MP} / \mathrm{TF}$-positive samples were associated with Pgp overexpression on their surface. Conversely, in the remaining samples $(n=30)$, treatment with the anti-FVIIa antibody did not significantly lengthen the time to clotting $(<10 \%)$, and Pgp overexpression was not detected. In addition, in the control
\end{abstract}

Correspondence to: Dr Antonio Angelini, Research Center on Aging and Translational Medicine (Ce.S.I. Met), 'G' d'Annunzio' University of Chieti-Pescara, 31 Via dei Vestini, I-66013 Chieti, Italy E-mail: aangelini@unich.it

*Contributed equally

Key words: multidrug resistance, P-glycoprotein, tissue factor, microparticles samples from healthy individuals, Pgp expression at the plasma membrane and clotting in the presence of the anti-FVII antibody were not observed, indicating the absence of MPs. The present study demonstrated that MPs in the blood of cancer patients promoted fibrin generation via TF/FVII-dependent pathways, thus suggesting that the evaluation of MP-TF activity may have a predictive value for Pgp-mediated MDR in various cancer types. Although further studies are required, the measurement of plasma MP-associated TF activity as a predictive biomarker may provide novel therapeutic perspectives to improve the prognosis and effectiveness of anti-cancer drugs in patients who are at a high-risk of Pgp-mediated MDR.

\section{Introduction}

Multidrug resistance (MDR) is a significant obstacle to successful cancer chemotherapy in numerous types of human tumour $(1,2)$. MDR occurs predominantly as a result of overexpression of the transmembrane permeability glycoprotein (Pgp) encoded by the MDR protein 1 gene (3). Pgp is a member of the ATP-binding cassette transporter family that, in resistant cancer cells, has a role as an efflux pump for various structurally and functionally unrelated chemotherapeutic drugs, including the anthracyclines, vinca alkaloids and taxans, thereby reducing their concentration in the cell cytoplasm and limiting the effectiveness of cancer chemotherapy (4-7).

Venous thromboembolic events represent an important complication in patients with cancer due to haemostatic system activation caused by aggressive chemotherapy (8-11). Furthermore, D-dimer levels, which are used as coagulation markers in cancer patients, have been observed to be higher in advanced disease and decreased in patients in post-treatment remission, which suggests that pro-coagulant activity in cancer patients could predict response or disease progression (12). The expression of tissue factor (TF), which is a transmembrane glycoprotein and the major cellular initiator of blood coagulation in vivo, on the surface of neoplastic cells varies across different cell types (13-15). TF binds plasma factor II/VIIa (FII/FVIIa) to form a bimolecular complex that actives factor X and factor IX, leading to the generation of fibrin $(16,17)$. Hypercoagulability 
has been associated with chemoresistance, and TF may have a role in this association $(16,17)$. In addition, previous studies reported that Pgp and TF tend to be coexpressed in numerous types of neoplastic cell, potentially in response to common stimuli such as chemotherapy (18). Therefore, evaluation of TF-dependent coagulation activity associated with the presence of TF on the surface of tumour cells may act as a predictive biomarker for the indirect identification of Pgp-mediated MDR in cancer patients.

In vitro and in vivo studies have demonstrated that malignant cells release a large number of microscopic cell membrane-derived vesicles, which are 0.1-1.0 $\mu \mathrm{m}$ in diameter and called microparticles (MPs), in response to chemotherapy or stimulation/induction of apoptosis (19). MPs carry various surface proteins that are characteristic of their parental cells (20). In addition, clinical studies have reported that TF is exposed on the surface of circulating MPs from patients with cancer, and that high levels of MP-associated TF activity in the plasma of cancer patients predicted an increased risk for thrombosis and poor prognosis (21-25). The evaluation of circulating MP-associated TF activity in cancer patients during chemotherapy could be used to predict thrombosis and the development of MDR. Therefore, this analysis, in association with tumour markers or biopsies, could have a prognostic value for cancer patients.

The present study aimed to investigate whether the MPs released by the plasma membrane of cancer cells during chemotherapy showed high levels of Pgp and TF coexpression on their surface, and whether a rise in circulating MPs coexpressing Pgp and TF may be indirectly predictive for the development of MDR and thromboembolic complications. MPs were isolated from the blood of 50 patients with a variety of malignant tumours who were receiving cancer chemotherapy, and were analysed for TF activity and Pgp overexpression. The results of this analysis were compared with those obtained for 10 healthy volunteers matched for age and gender, who were considered as negative controls.

\section{Materials and methods}

Reagents and antibodies. The murine anti-human cluster of differentiation 243 (CD243) monoclonal antibody (clone, UIC2; IgG2a; dilution, 1:20; catalog no., MCA2671A488) that recognizes an extracellular conformational epitope of Pgp was purchased from Bio-Rad Laboratories, Inc. (Hemel Hempstead, UK). The rabbit anti-human FVII polyclonal antibody (clone CLBVII-I; IgG1; dilution, 1:100; catalog no., MW1899) was obtained from Sanquin (Amsterdam, The Netherlands).

Collection of blood samples. The patients used in the present study were enrolled at the Department of Oncology, S.S. Annunziata Hospital (affiliated to 'G. d'Annunzio' University of Chieti-Pescara; Chieti, Italy). Between February 2012 and November 2014, 4 ml peripheral blood was collected from 50 cancer patients with solid tumours (including pancreatic, breast, gastrointesyinal and lung cancer) through venepuncture with a BD Vacutainer ${ }^{\circledR}$ blood collecting system (BD Biosciences, Franklin Lakes, NJ, USA), and placed into $4.5 \mathrm{ml}$ polypropylene tubes containing $3.8 \%$ sodium citrate. Whole blood samples were processed immediately at room temperature to inhibit cell activation. Blood samples were centrifuged at 3,000 x g for $20 \mathrm{~min}$ at room temperature in a swing-out rotor without break to isolate erythrocytes and platelets. Platelet-poor plasma (PPP) was then gently removed and coagulation tests were performed immediately. The remaining plasma was distributed in $250 \mu \mathrm{l}$ aliquots, snap frozen in liquid nitrogen and finally stored at $-80^{\circ} \mathrm{C}$ until use. Plasma samples from 10 healthy volunteers ( 5 female and 5 male; mean age, 43 years; range, 23-46 years) were similarly processed and assessed as negative controls. Informed consent was obtained from all patients, who received detailed information regarding the study, adhering to the Declaration of Helsinki.

Isolation of MPs from plasma samples. To isolate MPs, PPP obtained from whole blood was thawed at $37^{\circ} \mathrm{C}$, transferred to microtubes $(1.5 \mathrm{ml})$ and further centrifuged at $5,000 \mathrm{x} \mathrm{g}$ at room temperature for $10 \mathrm{~min}$ to remove residual platelets. Subsequently, PPP was centrifuged at $19,800 \mathrm{x}$ g for $15 \mathrm{~min}$ at $20^{\circ} \mathrm{C}$ with a fixed-angle, $45^{\circ}$ rotor (Centrifuge 5417R; Eppendorf, Hamburg, Germany) to obtain a fraction enriched in cell-derived MPs. Subsequently, MP-free plasma was removed from each tube and the resultant MP pellets (25-30 $\mu \mathrm{l})$ were washed twice in $\mathrm{Ca}^{2+} / \mathrm{Mg}^{2+}$-free phosphate-buffered saline (PBS), suspended in $0.3 \mathrm{ml} \mathrm{Ca}^{2+} / \mathrm{Mg}^{2+}$-free PBS and used immediately. $\mathrm{Ca}^{2+}$-free PBS solution was used to prevent fibrin generation in the solutions. Equal volumes $(250 \mu \mathrm{l})$ of plasma taken from healthy volunteers were run in parallel as controls.

Pro-coagulant activity of MP-associated TF. The pro-coagulant activity of TF associated with MPs was evaluated in the presence and absence of the anti-FVIIa antibody. The analysis was performed using a spectrophotometer microplate reader (Spectra Max 190; Molecular Devices, LLC, Sunnyvale, CA, USA) equipped to analyse kinetic data in a 96 -well format. The instrument consists of an incubator cabinet with a photometer. Data acquisition and analysis were performed with SoftMax ${ }^{\circledR}$ Pro 4.0 software (Molecular Devices, LLC). Fresh PPP (90 $\mu \mathrm{l})$ was pipetted into four wells of a 96-well plate. Two wells were incubated with $3 \mu \mathrm{l}$ anti-human FVIIa antibody $(1 \mathrm{mg} / \mathrm{ml})$ to inhibit TF-mediated coagulation, while the remaining two wells were incubated with $3 \mu$ l physiological saline solution. The 96-well plate was transferred into the automated microplate reader and incubated at $37^{\circ} \mathrm{C}$ for $5 \mathrm{~min}$. Clotting was initiated by simultaneous addition to each well of $15 \mu \mathrm{l}$ of $0.1 \mathrm{M} \mathrm{CaCl}_{2}$ reagent pre-warmed at $37^{\circ} \mathrm{C}$. Following $5 \mathrm{sec}$ of agitation, fibrin formation was monitored by measuring the optical density (OD) of the plasma at $1 \mathrm{~min}$ intervals for $30 \mathrm{~min}$ at $405 \mathrm{~nm}$. Equal volumes of plasma taken from 10 healthy volunteers were run in parallel as controls. A plot of OD versus time was generated, and the half-maximal value (1/2 Vmax) of the OD change during clotting was determined and expressed as the anti-FVII antibody-induced lengthening of the clotting time, as compared with the saline samples. The following formula was used: Lengthening time $(\%)=($ anti-FVII clotting time - saline clotting time/saline clotting time) x 100 . The clotting time obtained for the same re-calcified plasma $(20 \mathrm{mmol} / \mathrm{l}$ $\left.\mathrm{CaCl}_{2}\right)$ in the presence and absence of excess $(1 \mathrm{mg} / \mathrm{ml})$ rabbit anti-human FVII IgG polyclonal antibody was compared to establish the TF-FVII-dependence of the fibrin generation. The lag time of fibrin generation was defined as the time interval required for the solution turbidity to reach the $1 / 2 \mathrm{Vmax}$. 
Immunohistochemical analysis of Pgp expression on MPs. For the evaluation of Pgp expression, MP pellets were prepared from 50 cancer patients. Briefly, plasma containing MPs was centrifuged at $4,200 \times \mathrm{g}$ at room temperature for $10 \mathrm{~min}$ and the MP pellet was cytocentrifuged at $400 \mathrm{x} \mathrm{g}$ at room temperature for 10 min (Shandon CytoSpin 2 Cytocentrifuge; Thermo Fisher Scientific, Inc., Waltham, MA, USA). To assess MP samples for CD243 expression, the Histostain ${ }^{\circledR}$-Plus 112 kit was used (Invitrogen; Thermo Fisher Scientific, Inc.), according to the manufacturer's protocol. Briefly, slides were fixed for $10 \mathrm{~min}$ at room temperature with $3 \%$ paraformaldehyde in Dulbecco's $1 \mathrm{X}$ PBS (pH 7.6). To prevent non-specific binding, slides were incubated for $10 \mathrm{~min}$ with serum blocking solution, followed by incubation for $45 \mathrm{~min}$ at room temperature with murine anti-CD243 monoclonal antibody (1:20). Following washing three times with $1 \mathrm{X}$ PBS, slides were incubated for $10 \mathrm{~min}$ with the enzyme conjugate provided in the kit. Following further washing with PBS, samples were incubated for 10 min with 3,3'-diaminobenzidine, washed with distilled water and coverslipped with mounting medium. Subsequently, the slides were observed under a Zeiss Axioscope light microscope (Carl Zeiss AG, Oberkochen, Germany) equipped with a CoolSNAP camera and the MetaMorph Image Analysis system (Universal Imaging Corp, Downingtown, PA, USA).

Statistical analysis. Data are expressed as the mean \pm standard deviation and were analysed for statistical significance using the Student's t-test, with Excel 2011 (Microsoft Corporation, Redmond, WA, USA). $\mathrm{P}<0.05$ was considered to indicate a statistically significant difference.

\section{Results}

Clotting time assay or fibrin generation assay. The present study analysed 50 PPP samples from cancer patients, of which 20 samples showed high levels of MP-associated TF activity, as demonstrated by the clotting time measurements. As is shown in a representative curve obtained using plasma from one healthy subject (Fig. 1A), no clotting was observed in the presence or absence of an anti-FVIIa antibody when plasma was re-calcified to induce coagulation for at least $1,800 \mathrm{sec}$. In 30 of the 50 plasma samples, the addition of anti-FVIIa antibody did not significantly prolong the clotting time; the percentage of lengthening between the two curves was $<10 \%$ (Fig. 1B). However, in 20 of the 50 plasma samples, the time interval for the solution turbidity to reach the $1 / 2 \mathrm{Vmax}$ was significantly different between samples treated with saline and anti-FVIIa antibody $(\mathrm{P}=0.01)$. Fig. $1 \mathrm{C}$ shows a representative microplate assay curve obtained using plasma incubated with or without anti-FVII antibody, and demonstrates a time delay of thrombin generation (427.1 vs. $609.9 \mathrm{sec}$, respectively). Therefore, the addition of anti-FVIIa antibody induced a significant lengthening of clotting time $(\sim 43 \%$; $\mathrm{P}=0.02)$, indicating the presence of a high amount of MP-associated TF within the plasma sample. Generally, the treatment of 20 TF-positive samples with an anti-FVIIa antibody induced a significant lengthening of clotting time compared with the controls treated with saline alone $(700 \pm 280$ and $950 \pm 350 \mathrm{sec}$, respectively; $\mathrm{P}=0.01$; Fig. 2).

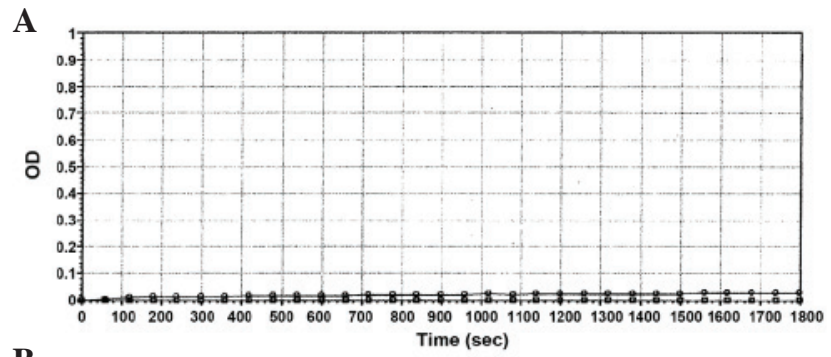

B

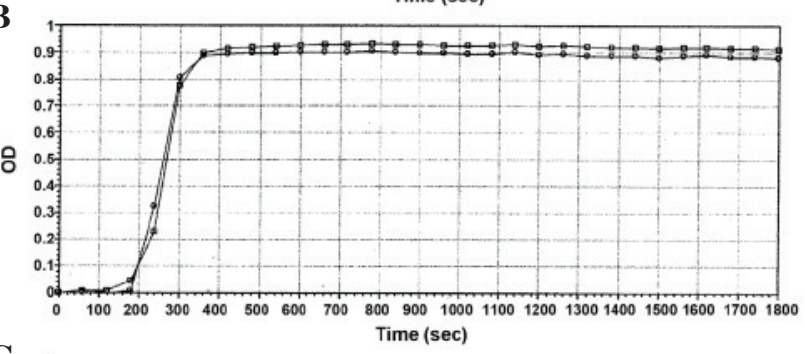

C

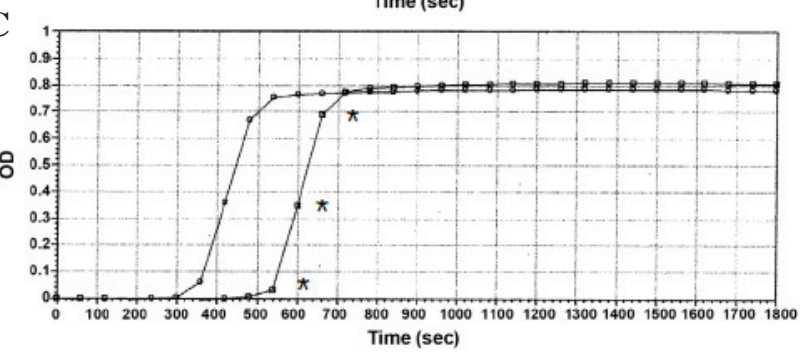

Figure 1. Coagulation rate of human plasma containing MPs from 50 cancer patients or 10 healthy volunteers. Plasma samples were incubated with saline or anti-FVII antibody. Coagulation was initiated by the addition of calcium chloride $(\mathrm{t}=0)$ and monitored for $1,800 \mathrm{sec}$. (A) No clotting was observed when plasma samples from healthy subjects were re-calcified in the presence or absence of anti-FVII antibody. The graph refers to one single experiment and is representative of 10 other consistent independent experiments. (B) For 30 cancer patients, the addition of an anti-FVII antibody to the plasma samples did not significantly affect $(\mathrm{P}>0.05)$ the clotting time compared with samples treated with saline alone. The graph displayed refers to a single experiment and is representative of 30 other consistent independent experiments. (C) For 20 cancer patients, the presence of an anti-FVII antibody in plasma samples induced a significant $\left({ }^{*} \mathrm{P}<0.05\right)$ lengthening $(\sim 43 \%)$ of the plasma clotting time compared with the control, which indicated the presence of tissue factor on the surface of MPs. The graph refers to a single experiment and is representative of 20 other consistent independent experiments. OD, optical density; MPs, microparticles; FVII, factor VII.

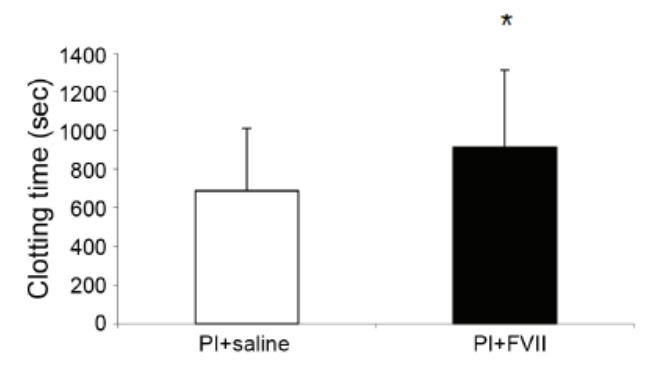

Figure 2. Clotting time in cancer patients. Comparison of the clotting times of 20 tissue factor-positive $\mathrm{Pl}$ samples from 20 cancer patients treated with saline $(700 \pm 280 \mathrm{sec})$ or an anti-FVII antibody $(950 \pm 350 \mathrm{sec})$. Treatment with anti-FVII antibody significantly lengthened $\left({ }^{*} \mathrm{P}<0.05\right)$ the time to coagulation initiation, as compared with the saline-treated control. Pl, plasma; FVII, factor VII.

Pgp expression on the surface of MPs. In order to determine whether TF and Pgp were coexpressed on the surface of MPs, Pgp expression on MPs isolated from plasma samples with a 
A

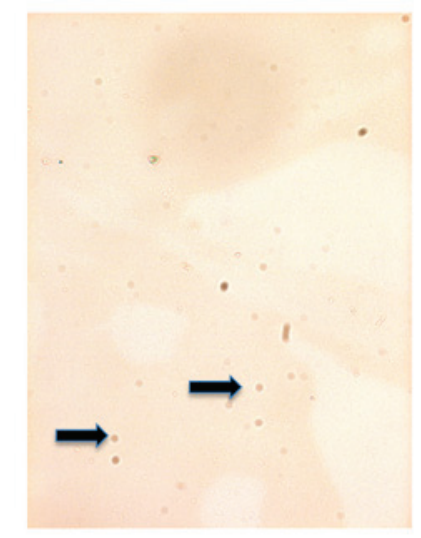

B

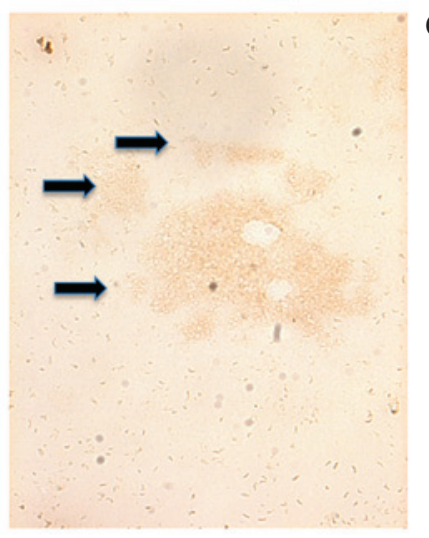

C

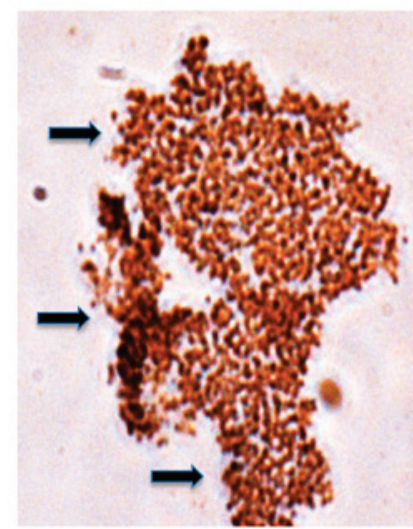

Figure 3. Immunohistochemical detection of permeability glycoprotein expression in cytocentrifuged microparticles from human plasma (magnification, $\mathrm{x} 40$ ). (A) There were few MPs in the plasma from healthy patients, and those MPs were negative for Pgp expression. (B) Numerous, clustered MPs were detected in the plasma samples from 30 cancer patients without TF activity, although these MPs showed very low expression of Pgp. (C) Numerous, clustered MPs were detected in the plasma samples from 20 cancer patients with elevated TF activity, and these MPs showed a high expression of Pgp, as indicated by the intense brown colouration. Black arrows indicate MPs and clusters of MPs. Pgp, permeability glycoprotein; MPs, micrcoparticles; TF, tissue factor.

significant lengthening of clotting time following inhibition of TF activity with an anti-FVIIa antibody was evaluated. MPs obtained from cancer patients that showed TF/FVII-dependent coagulation were compared with MPs from plasma samples with $<10 \%$ mean lengthening and MPs from plasma samples of healthy controls. As is shown in Fig. 3A, MPs were rarely detected in the plasma samples of healthy controls, and those that were detected were negative for Pgp expression. Similarly, very low or absent expression of Pgp was detected for the MPs isolated from plasma samples with no significant TF activity (lengthening, <10\%) (Fig. 3B). Conversely, a high number of MPs were strongly labelled for Pgp expression in plasma samples from cancer patients showing TF positivity (Fig. 3C).

\section{Discussion}

The present study demonstrated that Pgp and TF glycoproteins were associated with circulating tumour cell-derived MPs in blood collected from cancer patients undergoing chemotherapy. TF expression was identified on the surface of MPs by evaluating the TF-mediated pro-coagulant activity of plasma samples from 50 cancer patients by means of an anti-FVIIa antibody to distinguish fibrin generation via TF-FVII-dependent pathways. It was demonstrated that 20 of the 50 cancer patients $(>30 \%)$ had high levels of circulating MP-associated TF activity. Notably, treatment of these 20 plasma samples with an anti-FVIIa antibody significantly prolonged $(\mathrm{P}<0.05)$ the clotting time of these patients by up to $36.5 \pm 16 \%$, as compared with the clotting time of the same samples treated with saline alone. These results suggested that tumour cell-derived MPs contribute to the activation of coagulation in a TF-dependent manner, and indicated the specificity of this assay to inhibit the TF-dependent pro-coagulant activity via the FVII pathway. Conversely, in the remaining plasma samples that were negative for MP-associated TF $(n=30)$, the clot reaction curves of plasma samples treated with anti-FVIIa antibody were similar to those obtained following treatment of plasma samples with saline alone. These results suggested that the clotting times were unaffected by anti-FVIIa treatment, and that fibrin generation was independent of this factor.
Therefore, in these samples, anti-FVII treatment did not prolong the fibrin generation. Furthermore, the total absence of coagulation in the human plasma obtained from healthy individuals incubated with anti-FVIIa antibody suggested the absence of MPs or the presence of very low levels of MPs in the blood of healthy subjects, as compared with the plasma samples of cancer patients.

These results were consistent with the analysis of the expression of $\mathrm{Pgp}$ on the surface of cell-derived MPs. Notably, high levels of Pgp-positive MPs were observed in the 20 samples that showed high TF pro-coagulant activity. Conversely, in the TF-negative samples, the MPs were lower in number and showed a reduced fibrin-generating capacity, as compared with the TF- and Pgp-positive MPs. No coagulant activity and Pgp expression was observed in plasma samples from healthy individuals. These results indicated that the TF activity was significantly increased on the surface of circulating MPs derived from the blood of 20 cancer patients undergoing chemotherapy, and that this was closely associated with Pgp overexpression. This is consistent with previous studies that demonstrated that high levels of MP-associated TF activity were associated with various types of tumour, including breast cancer and pancreatic adenocarcinoma, and with a poor response to chemotherapy (26-31). Therefore, the analysis of MP-associated TF activity could be used as a potential positive predictor for Pgp-mediated MDR, thereby generating novel avenues for assessing MDR in cancer patients during chemotherapy.

The present study demonstrated that, in cancer patients undergoing chemotherapy, the measurement of MP-associated TF pro-coagulant activity using a rapid and simple turbidimetric assay may represent an important strategy for the identification of patients with a potential Pgp-mediated MDR phenotype. Such a strategy may permit the collation of useful information on MDR progression, and allow early intervention with novel combinations of anticancer drugs, including those that are structurally modified analogues of MDR-associated agents so as to avoid unnecessary therapy, or Pgp inhibitors to inhibit or modulate MDR development $(32,33)$. Therefore, TF-positive MPs may be considered useful biomarkers for the 
prediction of therapeutic response and for the development of personalized therapeutic strategies for cancer patients.

In addition, the analysis of MP-associated TF activity could be used alongside other markers of coagulation, including prothrombin time and activated partial thromboplastin time, to provide additional information on the pro-coagulant potential of MPs released from cancer cells and to eventually develop an effective and safe anti-thrombotic therapy.

In conclusion, the present study demonstrated that there was an association between TF-activity and Pgp expression on the surface of circulating MPs in cancer patients, which suggested that the levels of TF-positive MPs may be a useful predictive biomarker for the identification of patients with an increased risk for MDR, and to monitor the response to targeted therapeutics. However, these observations require verification in a larger study population, in which cut-off values for TF-positive MP activity may be elucidated. The results of the present study provided a rationale for assessing alternative therapeutic strategies for cancer patients with high plasma levels of TF-positive MPs, in order to overcome Pgp-mediated MDR.

\section{References}

1. Ling V: Multidrug resistance: Molecular mechanism and clinical relevance. Cancer Chemother Pharmacol 40 (Suppl): S3-S8, 1997.

2. Gottesman MM: Mechanisms of cancer drug resistance. Annu Rev Med 53: 615-627, 2002.

3. Sauna ZE, Smith MM, Müller M, Kerr KM and Ambudkar SV: The mechanism of action of multigrug-resistance-linked P-glycoprotein. J Bioenerg Biomembr 33: 481-491, 2001.

4. Gottesmann MM, Fojo T and Bates SE: Multidrug resistance in cancer: Role of ATP-dependent transporters. Nat Rev Cancer 2: 48-58, 2002.

5. Gillet JP and Gottesman MM: Mechanisms of multidrug resistance in cancer. Methods Mol Biol 596: 47-76, 2010.

6. Higgins CF: Multiple molecular mechanism for multidrug resistance transporters. Nature 446: 749-757, 2007.

7. Ward AB, Szewczyk P, Grimard V, Lee CW, Martinez L, Doshi R, Caya A, Villaluz M, Pardon E, Cregger C, et al: Structures of $\mathrm{P}$-glycoprotein reveal its conformational flexibility and an epitope on the nucleotide-binding domain. Proc Natl Acad Sci USA 110: 13386-13391, 2013

8. Lyman GH, Khorana AA, Kuderer NM, Lee AY, Arcelus JI, Balaban EP, Clarke JM, Flowers CR, Francis CW, Gates LE, et al; American Society of Clinical Oncology Practice: Venous thromboembolism prophilaxis and treatment in patients with cancer: American Society of Clinical Oncology clinical practice guideline update. J Clin Oncol 31: 2189-2204, 2013.

9. Sørensen HT, Mellemkjaer L, Olsen JH and Baron JA: Prognosis of cancers associated with venous thromboembolism. N Engl J Med 343: 1846-1850, 2000.

10. Heit JA, Silverstein MD, Mohr DN, Petterson TM, O'Fallon WM and Melton LJ III: Risk factors for deep vein thrombosis and pulmonary embolism: A population based case-control study. Arch Intern Med 160: 809-815, 2000.

11. Khorana AA, Francis CW, Culakova E, Kuderer NM and Lyman GH: Frequency, risk factors, and trends for venous thromboembolism among hospitalized cancer patients. Cancer 110 : 2339-2346, 2007.

12. Beer JH, Haeberli A, Vogt A, Woodtli K, Henkel E, Furrer T and Fey MF: Coagulation markers predict survival in cancer patients. Thromb Haemost 88: 745-749, 2002.

13. Callader NS, Varki N and Rao LV: Immunohistochemical identification of tissue factor in solid tumors. Cancer 70: 1194-1201, 1992.

14. Kataoka H, Uchino H, Asada Y, Hatakeyama K, Nabeshima K, Sumivoshi A and Koono M: Analysis of tissue factor and tissue factor pathway inhibitor expression in humanoid colorectal carcinoma cell lines and metastatic sublines to the liver. Int J Cancer 72: 878-884, 1997.
15. Uno K, Homma S, Satoh T, Nakanishi K, Abe D, Matsumoto K, Oki A, Tsunoda H, Yamaguchi I, Nagasawa T, et al: Tissue factor expression as a possible determinant of thromboembolism in ovarian cancer. Br J Cancer 96: 290-295, 2007.

16. Antoniou D, Pavlakou G, Stathopoulos GP, Karvdis I, Chondrou E, Papageorgiou C, Dariotaki F, Chaimala D and Veslemes M: Predictive value of D-dimer plasma levels in response and progressive disease in patients with lung cancer. Lung Cancer 53: 205-210, 2006.

17. Tomimaru Y, Yano M, Takachi K, Kishi K, Miyashiro I, Ohue M, Ohigashi H, Sasaki Y, Ishikawa O and Imaoka S: Correlation between pretreatment d-dimer levels and response to neoadjuvant chemotherapy in patients with advanced esophageal cancer. Dis Esophagus 21: 281-287, 2008.

18. Lwaleed BA and Cooper AJ: Tissue factor expression and multidrug resistance in cancer: Two aspects of a common cellular response to a hostile milieu. Med Hypotheses 55: 470-473, 2000.

19. Piccin A, Murphy WG and Smith OP: Circulating microparticles: Pathophysiology and clinical implications. Blood Rev 21: 157-171, 2007.

20. Hugel B, Martínez MC, Kunzelmann C and Freyssinet JM: Membrane microparticles: Two sides of the coin. Physiology (Bethesda) 20: 22-27, 2005.

21. Haubold K, Rink M, Spath B, Friedrich M, Chun FK, Marx G, Amirkhosravi A, Francis JL, Bokemeyer C, Eifrig B and Langer F: Tissue factor procoagulant activity of plasma microparticles is increased in patients with early-stage prostate cancer. Thromb Haemost 101: 1147-1155, 2009.

22. Hron G, Kollars M, Weber H, Sagaster V, Quehenberger P, Eichinger S, Kyrle PA and Welterman A: Tissue factor-positive microparticles: Cellular origin and association with coagulation activation in patients with colorectal cancer. Thromb Haemost 97: 119-123, 2007.

23. Zwicker JI, Kos CA, Johnston KA, Liebman HA, Furie BC and Furie B: OC-06 tissue factor-bearing microparticles are associated with an increased risk of venous thromboembolic events in cancer patients. Thromb Res 120 (Suppl): S143, 2007.

24. Zwicker JI, Liebman HA, Neuberg D, Lacroix R, Bauer KA, Furie BC and Furie B: Tumor-derived tissue factor-bearing microparticles are associated with venous thromboembolic events in malignancy. Clin Cancer Res 15: 6830-6840, 2009.

25. Manly DA, Wang J, Glover SL, Kasthuri R, Liebman HA, Key NS and Mackman N: Increased microparticle tissue factor activity in cancer patients with venous thromboembolism. Thromb Res 125: 511-512, 2010.

26. Lechner D and Weltermann A: Circulating tissue factor-exposing microparticules. Thromb Res 122 (Suppl 1): S42-S54, 2008.

27. Thaler J, Ay C, Mackman N, Bertina RM, Kaider A, Marosi C, Key NS, Barcel DA, Scheithauer W, Kornek G, et al: Microparticle-associated tissue factor activity, venous thromboembolism and mortality in pancreatic, gastric, colorectal and brain cancer patients. J Thromb Haemost 10: 1363-1370, 2012.

28. Thaler J, Ay C, Mackman N, Metz-Schimmerl S, Stift J, Kaider A, Müllauer L, Gnant M, Scheithauer W and Pabinger I: Microparticle-associated tissue factor activity in patients with pancreatic cancer: Correlation with clinicopathological features. Eur J Clin Invest 43: 277-285, 2013.

29. Bharthuar A, Khorana AA, Hutson A, Wang JG, Key NS, Mackman $\mathrm{N}$ and Iyer R: Circulating microparticle tissue factor, thromboembolism and survival in pancreaticobiliary cancers. Thromb Res 132: 180-184, 2013.

30. Tesselaar ME, Romijn FP, Van Der Linden IK, Prins FA, Bertina RM and Osanto S: Microparticle-associated tissue factor activity: A link between cancer and thrombosis? J Thromb Haemost 5: 520-527, 2007.

31. Nitori N, Ino Y, Nakanishi Y, Yamada T, Honda K, Yanagihara K, Kosuge T, Kanai Y, Kitajima M and Hirohashi S: Prognostic significance of tissue factor in pancreatic ductal adenocarcinoma. Clin Cancer Res 11: 2531-2539, 2005.

32. Angelini A, Di Pietro R, Centurione L, Castellani ML, Conti P, Porreca E and Cuccurullo F: Inhibition of P-glycoprotein-mediated transport by $\mathrm{S}$-adenosylmethionine and cynarin in multidrug-resistant human uterine sarcoma MES-SA/Dx5 cells. J Biol Regul Homeost Agents 26: 495-504, 2012.

33. Angelini A, Centurione L, Sancilio S, Castellani ML, Conti P, Di Ilio C, Porreca E, Cuccurullo F and Di Pietro R: The effect of the plasticizer diethylhexyl phthalate on transport activity and expression of P-glycoprotein in parental and doxo-resistant human sarcoma cell lines. J Biol Regul Homeost Agents 25: 203-211, 2011. 